\title{
SOME OBSERVATIONS ON THE EFFECT OF CENTRIFUGAL FORCE IN MAN
}

\author{
BY \\ Squadron-Leader W. K. STEWART, A.F.C., R.A.F. (V.R.) \\ From the R.A.F. Institute of Aviation Medicine
}

(Received June, 1945)

\begin{abstract}
ANY flight manouvre, whether simple or complex, imposes varying degrees or types of acceleration upon the aircraft and the crew. Centripetal acceleration is developed when an aircraft is moving in a curved path, and its value is directly proportional to the square of the angular velocity. For a constant indicated air-speed, the centripetal acceleration is inversely proportional to the radius of curvature of the flight path. It follows from Newton's First Law of Motion that a force must be acting upon the aircraft and directed normally towards the centre of curvature, but on account of inertia, the aircraft resists this force; the line of action of this resistance is opposite to the centripetal force, is therefore directed away from the centre and is termed centrifugal force. If the pilot be regarded as an inanimate part of the aircraft, it is convenient to think in terms of this centrifugal force, which presses him into his seat and is responsible for the physiological effects.
\end{abstract}

It is also convenient to express the value of centrifugal force $(\mathrm{G})$ as multiples of gravity by the following formula:

where

$$
\mathrm{G}=\frac{\mathrm{V}^{2}}{15 \mathrm{r}} \cdot \mathrm{g} \text { (gravity units) }
$$$$
\mathrm{V}=\text { indicated speed in m.p.h. }
$$$$
\mathrm{r}=\text { radius of turn in feet. }
$$

In practice, this formula is of little value since the force acting upon the pilot is the resultant of the centrifugal force and gravity, and is measured directly by an accelerometer.

The sensations attendant upon exposure to centrifugal forces of any magnitude have been described graphically and in some detail by Rook and Dawson (1938), Livingston (1939), Diringshofen (1938 and 1939). The latter author and his collaborators have investigated many aspects of the problem and as a result of their basic researches, it can be stated that the more severe effects are due to a fall in blood pressure in the carotid arteries. This causes the loss of vision known as " Blacking Out " amongst fighter pilots and also the loss of consciousness, which, however, is infrequent in occurrence. It follows that any practical method, by which the fall in blood pressure can be prevented, also aids the pilot in retaining vision. The importance of this to any pilot, during combat, need not be stressed. The fall in blood pressure in the carotid arteries is due to the line of action of the force passing vertically through the spine of a pilot in the sitting position. Not only has the heart to pump blood against this force, but the cardiac output also falls due to reduction in venous return and pooling of the blood in the abdomen and lower limbs.

An opportunity has been afforded to one subject (W.K.S.) to make frequent observations of the subjective effects, and an analysis of some of the objective effects, of centrifugal force in flight. This was achieved by the use of cine photography in a two-seater aircraft. Approximately 50 subjects have been observed by this method, but the following account deals principally with the correlation of the objective and subjective effects of the subject (W.K.S.).

The effects of these forces may be classified in three main phases: first, the general sensations of the apparent increase in weight of the body; secondly, the loss of vision or " blacking-out"; thirdly, the loss of consciousness which is infrequent and is usually preceded by a period of "black-out".

\section{General Sensations}

Somatic sensations are principally dependant upon the effective increase in weight. At $G$ loadings of over 5, movements of limbs in the plane of the centrifugal force either become impossible or extremely difficult according to the physical build of the individual. Movements in a plane at right angles to the force were still possible at these loadings but were comparatively slow and awkward in unsupported limbs. There may also be a significant increase in the latent period which may be partly central and partly peripheral in origin (Burmeister, 1939). Care must be taken in the interpretation of such experimental results since the line of axis of some of the muscles concerned in the movement may be in the plane of centrifugal force. Further common symptoms of the increase in weight were a downward drag of the facial tissues and the "concertina sensation" of the trunk. Objectively, the effects of these were easily seen in the deepening of 
the naso-labial folds, nasal ridges becoming more pronounced and in the development of subocular pouches, the lower lids sometimes showing a marked eversion (Figs. 1 and 2). If the erect posture were maintained, not only did the soft tissues become moulded but there also occurred an apparent de-

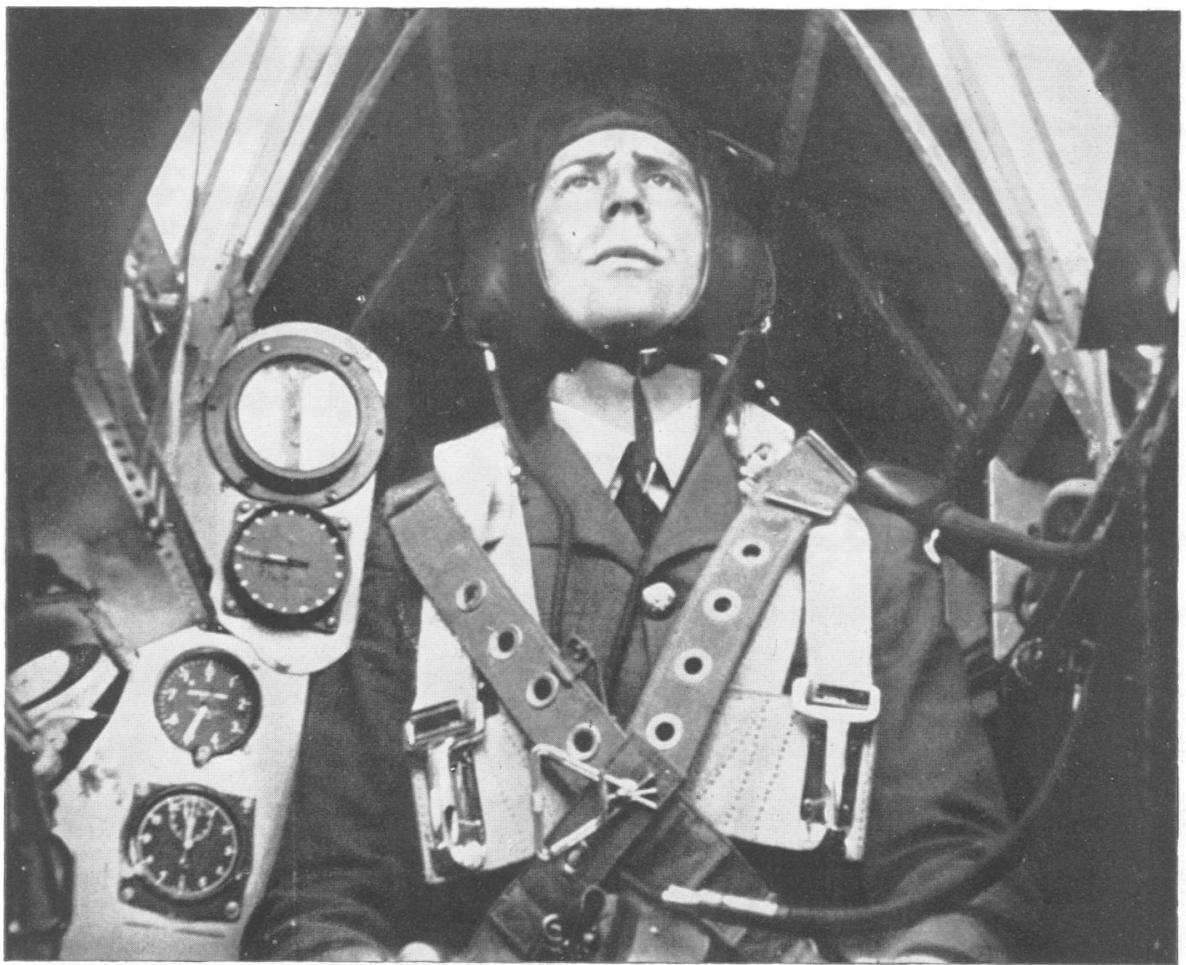

FIG. 1.-Subject seated in rear cockpit of Battle. Kollsman Accelerometer recording approximately 5 G.

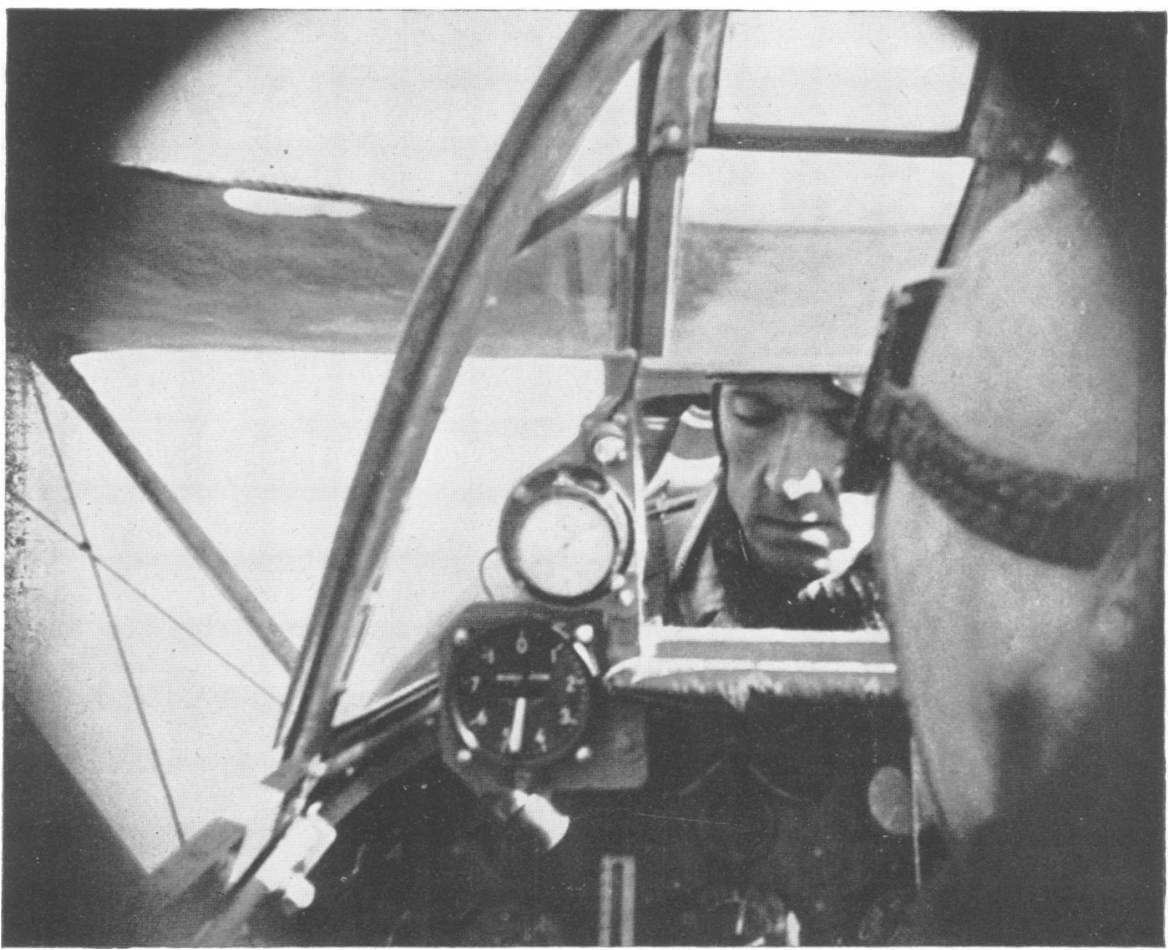

FIG. 2.-Subject in Gladiator. Kollsman Accelerometer recording approximately 5 G. 
crease in the height of the trunk of 1-2 inches, and if these effects were marked, the subject appeared to have acquired new features. Under heavy $G$, whether vision is affected or not, most subjects appear to have delayed cerebration, to be slightly dazed as if their spatial and temporal orientation are impaired.

\section{Visual Impairment-Blacking-out}

The above'reactions are of secondary importance to the disturbance of vision or of consciousness. It was found necessary, early in the course of the research, to standardize the recognition of the endpoint of the visual loss. Consequently a test-type card with letters of standard dimensions was hung in the cockpit before the observer, and above the camera level. Thus for all comparative work, the abolition of contrast between letters and background was taken as the commencement of the "black-out" period. At no time did distant vision remain after this had occurred, the amaurosis being complete. There was, however, considerable difficulty in analysing the sequence of events. When the testtype card was taken as the visual stimulus, the usual degrees of impairment could be differentiated easily. Slight "veiling" could be distinguished as a mere greying of the white background, the black letters still being distinct. Severe "veiling" could be differentiated as a further stage, when the card would become almost black and yet the central letters still be distinguished as a form of tunnel vision. The end-point was reached when the letters had disappeared, the general sensation being that of blackness. These stages usually succeeded one another, although, not infrequently, the first two stages seemed to be omitted, the onset of the " black-out" being extremely sudden. These experimental observations can be compared to the sequence of events in fighter aircraft. In the turret of a two-seater fighter, it was observed that according to the final value and rate of onset of $G$, distant vision became blurred and was lost before near vision. Objects subtending a small angle at the retina, such as details of the ground and distant aircraft, definitely disappeared before turret details such as the ring and spot of the reflector sight. "Veiling" of vision was generally associated also with darkening of the clouds, and blurring of cockpit detail. The final end-point of vision was of course similar in that everything became black as if the sensation of light were lost. Thus, little information could be derived from an analysis of the amaurosis, for although in most cases it was similar to the action of a diaphragm, yet often the classical German description of "Vorhang" applied. The onset was so rapid that the order of impairment of the upper and lower segments of the visual field could not be determined. Occasionally an amaurosis of rapid onset appeared not to give a sensation of "blacking-out" but of "whiting-out" - as if light were still appreciated. This has also been experienced by some pilots and is not uncommon. The whole of the visual field was affected; at no time was there any hemianopic or scotomatous impairment. Finally the return of vision was rapid. Intermediate stages could not be differentiated, nor were residual visual symptoms ever experienced.

\section{Oculomotor Reactions}

It has been pointed out before that general somatic sensation is not subjectively greatly impaired when "blacking-out" takes place and that coordinated movements are still possible. In general, the integrity of the external oculomotor muscles was found to be maintained during the period of " blackout " and full and co-ordinated ocular movements were possible in every direction. There did not appear to be any significant prolongation of the time taken to complete these movements when "blacked-out", as compared to the time taken normally. Fixity of gaze appeared to be a constant feature during the "black-out" period. This can be ascribed to the subject concentrating on timing as accurately as possible the duration of the visual loss. Three experiments were carried out during which the subject was " blacked-out" with his head turned strongly to the right but with gaze fixated on the test-type card. There was no deviation of gaze from the point of fixation even in an amaurosis lasting for 11-12 seconds.

During the course of the experiments, a left-sided esophoria had been observed under high loadings ( $5 \mathrm{G}$ or over). The degree of imbalance, though slight, was definite, occurred usually in the left eye and appeared to be peculiar to the individual (Fig. 3). It was most often associated with the development of the amaurosis, but also occurred before visual impairment was experienced, and also at high $G$ when the subject was protected from the circulatory effects. Once developed, the esophoria remained throughout the period of high $\mathrm{G}$, and on one occasion progressed to a stage almost of skew deviation. On another occasion the eyes were voluntarily moved whilst the esophoria was present. The visual axes were then seen to be readjusted-the imbalance disappearing during the movement and developing again in the period of rest.

Nystagmoid movements were never recorded, but. frequently at high values of $G$ there developed a considerable degree of ptosis of the right upper eyelid, not unlike a unilateral Horner's Syndrome. This was frequently associated with "blacking-out", but also developed apart from visual impairment. (Fig. 4). There was never any paresis of the facial musculature.

\section{Sensations associated with Loss of Vision}

Other sensory functions did not appear to be markedly impaired. At no time was there any actual loss of hearing as reported by Rook and Dawson (1938). If auditory acuity became impaired there was always an association with pressure effects. in the middle ear sustained during the preliminary dive. There was never any actual nausea, sickness, giddiness, or tinnitus during the application of high acceleration. Sensory somatic functions were also 


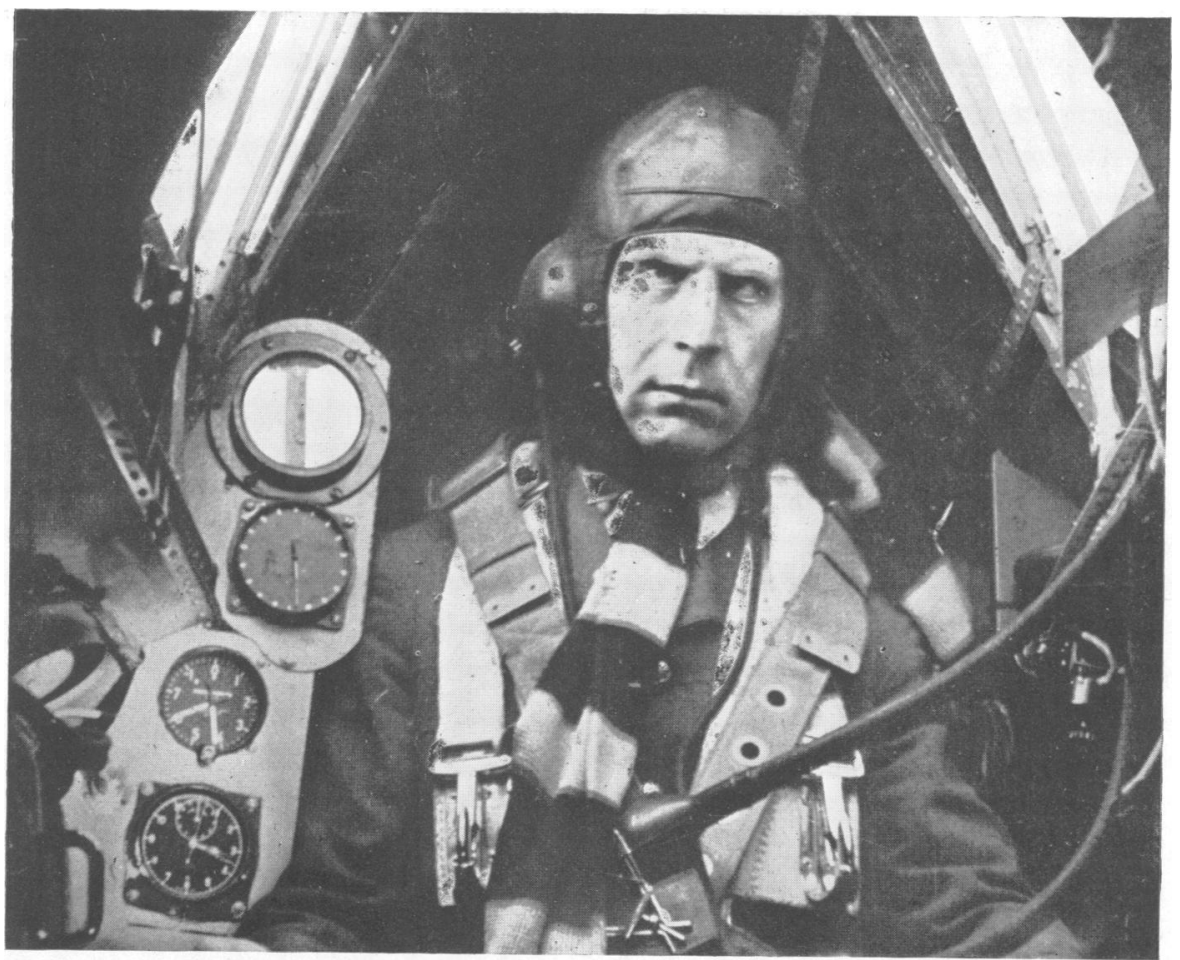

Fig. 3.-Esophoria of left eye in severe black-out. Acceleration value $4 \cdot 4$ G. (Maximum-6 G.)

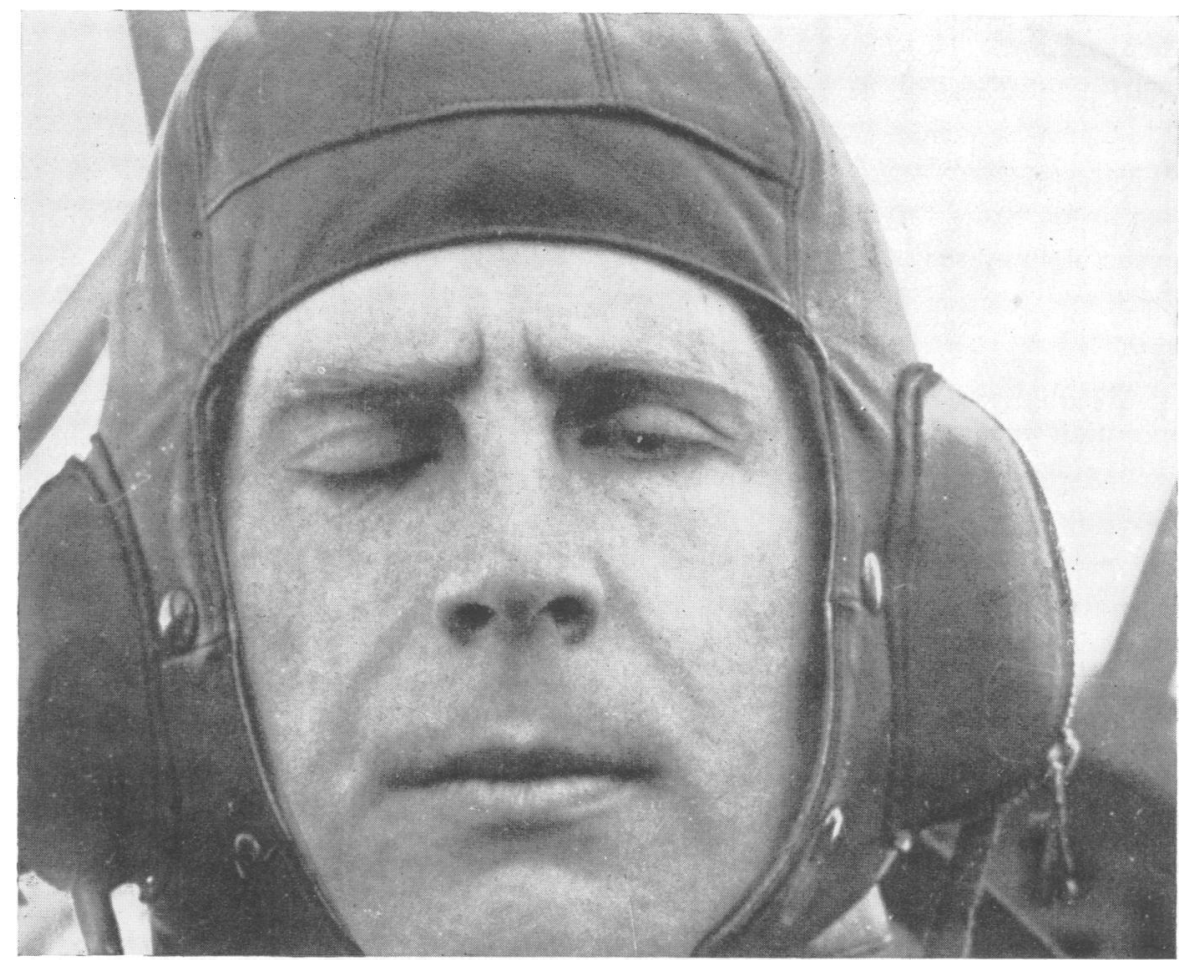

FIG. 4. - Ptosis of upper eyelids in blacked-out subject. Acceleration value 5.8 G. 
relatively unaffected-even in an amaurosis lasting as long as 12 seconds. Posture was normally maintained; limb positions were appreciated and movements of small amplitude were executed with ease. The sense of smell was found to be present when the subject was "blacked-out". Oil of cloves vapour delivered through a nasal catheter during a prolonged "black-out" was appreciated. Pain was never experienced. Headache of frontal type was occasionally experienced by the pilot, but its origin could not be dissociated from sinus pressure. An interesting sensation which was frequently recorded, was the occurrence of paræsthesia in the legs. This was of a tingling or burning character-localized to the calves and lateral aspects of the legs. It only occurred under heavy $G$ loadings and was unrelated to the actual "black-out", being experienced by the pilot who had complete retention of vision and also by the subject who had visual loss. These symptoms were absent when the circulation in the lower limbs was arrested by means of cuffs, and therefore may be due to hydrostatic distension of the vessels. However, analysis of sensations incurred with heavy loadings must at best be rudimentary-apart from the dominating sensation of the loss of vision. This caused some psychological upset, evidenced usually by apprehension often associated with an unpleasant feeling in the abdomen. Most subjects experienced apprehension in their first few " black-outs ", and it was noteworthy how disconcerting a " black-out" could be to pilots with many hundreds and thousands of flying hours to their credit, but who, owing to the nature of their work, had not been subjected to more than $3 \frac{1}{2}-4 \mathrm{G}$ for a considerable time. However, apprehension disappeared as the subject became accustomed to " blacking-out,".

\section{Removal of Acceleration}

The removal of $\mathrm{G}$ did not cause any symptoms. Just as the general increase in the weight of the limbs must cause some peripheral sensorimotor disturbance, likewise the removal of the force may have similar effects on the kinæsthetic judgment. It was frequently observed, for example, that the subject's conception of when the $G$ was finally removed and his subsequent behaviour, often coincided with an accelerometer reading of $2 \mathrm{G}$. In two of fifty subjects, when the aircraft was resuming level flight and the $G$ falling off, there was distinct spatial disorientation associated with a sense of diving steeply, almost as if the aircraft were turning on its back. Dizziness, nausea, and profuse sweating were also noticed. A complete "black-out" at $5 \frac{1}{4} \mathrm{G}$ in one of these subjects only resulted in slight discomfort, but again spatial disorientation was experienced, during removal of the $G$, and caused the subject to lean back in an effort to overcome the sensation of falling forward. Although postural improvement allowed the subject to sustain $5.5 \mathrm{G}$ without visual symptoms, it did not alleviate the dizziness and associated effects. It is interesting to note that this observer is prone to air-sickness,

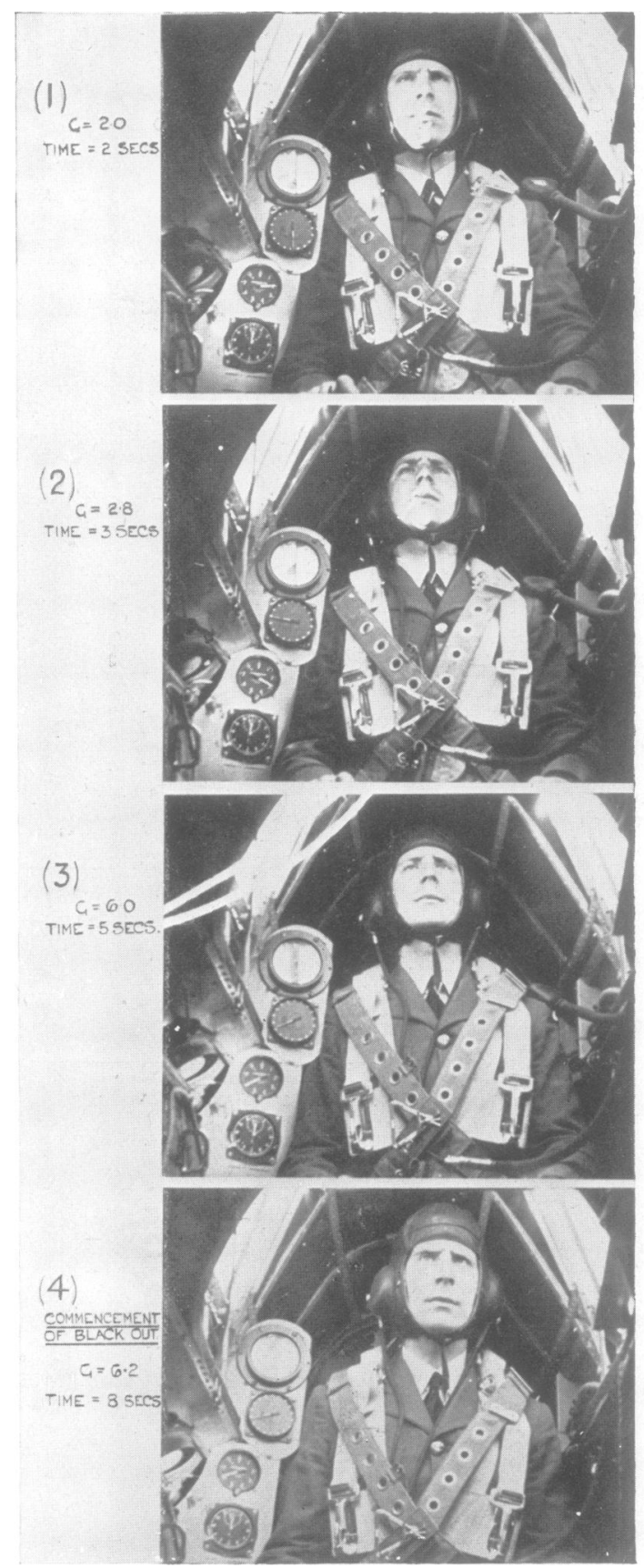

FIG. 5.-Onset and development of unconsciousness.

but at the time of these tests adaptation had taken place.

\section{After Effects of Blacking-Out}

Immediate sequelæ were not marked for W.K.S., but occasionally pilots reported some slight loss of spatial orientation in that, after "blacking-out" severely, a further slow circuit would have to be made in order to establish their surroundings. On completion of any flight, the most marked effect was the feeling of undue tiredness, lethargy, and fatigue. 

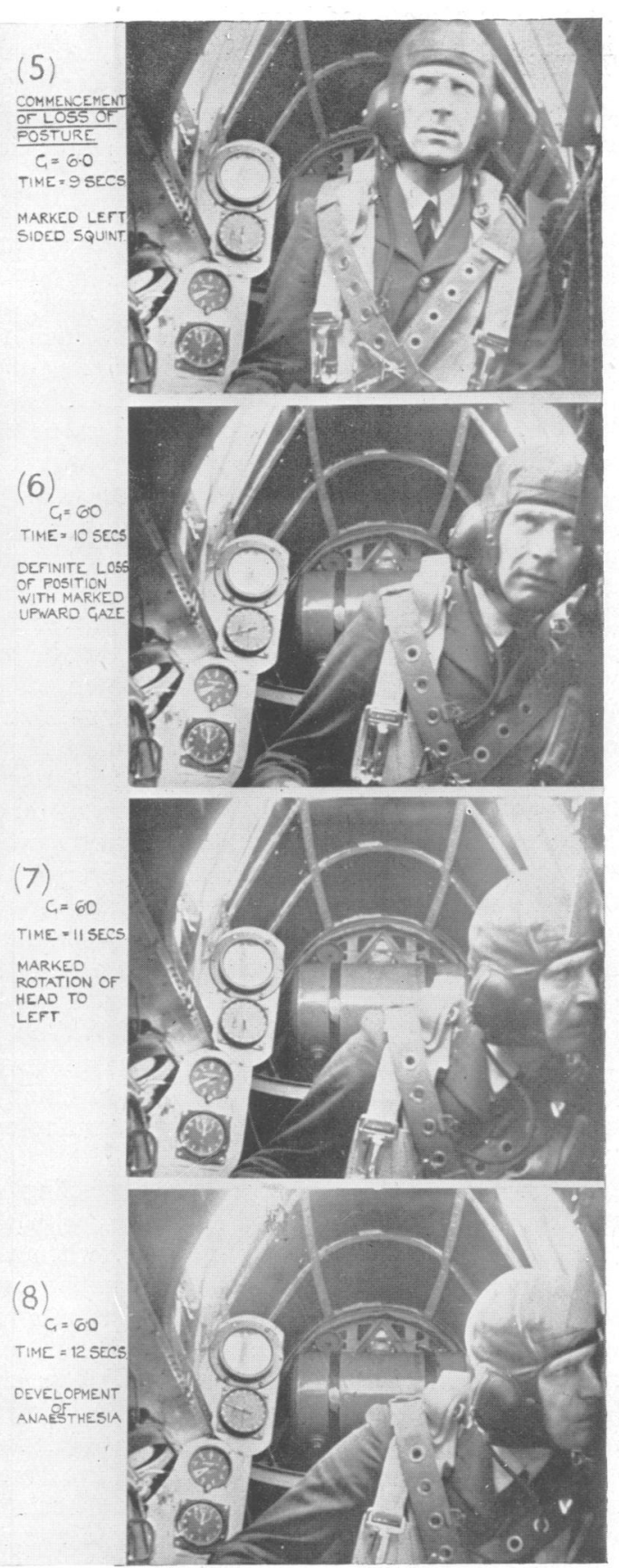
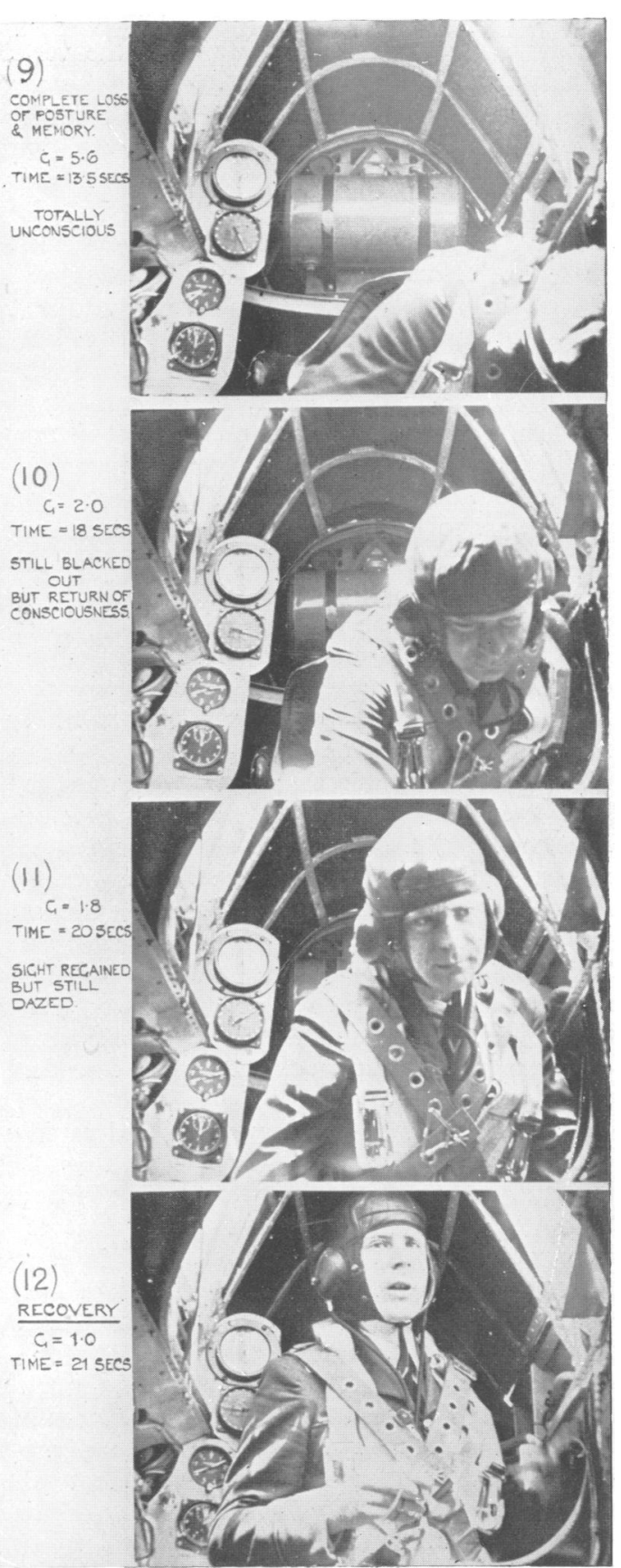

FIG. 5.-Onset and development of unconsciousness.

This was particularly noted in the lower limbspresumably because they were the first members to be exercised. In addition to this, however, power of concentration would be definitely diminished after 3-4 consecutive " black-outs "; memory also seemed to be impaired, and the subject frequently felt depressed and unsociable in the evening. Occasionally there was some disturbance of the sleep rhythm-early morning awakening being a feature. These effects were much more marked at the commencement of the period of research, but continued in some measure or other throughout the whole period-the fatigue and lethargy being especially persistent. No permanent impairment of eyesight occurred. A fact worthy of further investigation emerged in that after any period of intensive flying, the subject, who had slight heterophoria, definitely experienced headaches associated with occasional double vision. These symptoms were not experienced during periods in which no flying took place and had never been noted by the subject before. Pilots and subjects complained 
occasionally of backaches due to muscular strain. $\mathrm{X}$-rays revealed no abnormalities of the vertebral column. There were no effects upon the cardiovascular or nervous systems and no tendency to develop varicosities in veins of legs.

\section{Unconsciousness}

Total loss and impairment of consciousness of various degrees was experienced by W.K.S. on several occasions. The introduction of the term impairment is, of course, open to some objections, as it is based on the recording of motor reactions. It is firmly believed, however, that there is a gradient in the loss of function of nervous tissue which ranges from some lengthening of reaction time at subthreshold values of $G$, through the stage of visual symptoms, to the total abolition of all motor and sensory functions. This final stage is always associated with true amnesia and loss of consciousness. The criterion for cortical impairment could only be derived from the correlation of subjective effects with objective or automatic photographic recording, since no performance tests or other investigations could be applied.

Complete unconsciousness was only deemed to have been present when the following events were recorded: (1) Definite alteration in posture and motor reactions. (2) Loss of general sensibility and muscle tone. (3) Amnesia. (4) Subjective sensations greater than those experienced in simple "blacking-out". Summaries of two experiments illustrate these points. It must again be emphasized that this subjective and retrospective analysis must be regarded as being coloured with considerable psychological disturbance.

Complete "black-out" was achieved rapidly in one experimental flight. The subject then found himself lying on the floor of the cockpit in front of the camera, having fallen completely out of the harness, which at that time was a simple gunner's waist belt. There was no recollection of striking the floor, although both face and arms were considerably bruised. Sound was first appreciated, then pain and posture, and finally vision, though this may be partly due to the fact that illumination in the well of the aircraft was of low intensity. The principal emotion was fear, but nevertheless general psychological upset and disorientation were present -for example, fluid which had poured from the inverted nasal sinuses under the acceleration was initially thought to be cerebro-spinal fluid.

A second experiment is illustrated in Figs. 5 and 6. On both these occasions it did appear as if some degree of consciousness were recovered before full return of vision.

Impairment of consciousness was said to exist when there was: (1) No marked alteration in general posture but some loss in the motor reactions of the limbs and muscle tone. (2) Subjective sensations not experienced in "blacking-out". The difference between impairment and complete unconsciousness is more academic than practical since control of the aircraft would have been lost in either case, if the subject had been piloting. Impairment included some loss of somatic sensation particularly of the arms, associated with loss of muscular power, but amnesia was absent in that the subject did not recognize any marked change in his environment after vision returned.

The recognition of a period of unconsciousness is rarely attained unless it produces a marked change in environment. This, of course, is always produced in single-seater aircraft, due to loss of control; but under the experimental conditions, in which the subject, knowing that the procedure under investigation might produce unconsciousness, and taking the precaution of adjusting the safety harness tightly, recognition of unconsciousness could not always be definite. It has been found that impairment might occur without the subject being aware of more than a very severe " black-out". In one case, marked alteration in posture occurred, a subject falling forward and striking his nose on the intercommunication tubing without even being aware that he had " blacked-out". This was never experienced by W.K.S., a preceding stage of visual loss always occurring.

Loss of posture under centrifugal force is magnified by the increase in weight of the various body components. Any loss of muscular power may be greatly enhanced by the mechanical effects of the $G$ upon the part affected. One has also to be cautious of interpreting loss of posture on nonmedical or inexperienced observers. There may be some instinct present to yield to the acceleration by bending forwards particularly when "blacked-out". This can be complicated by the fact that any given pilot who may be undergoing acceleration tests for medical categorization may confuse the terminology " black-out" with loss of consciousness.

Apart from nausea and a general feeling of malaise, the symptoms varied somewhat with the experimental procedure. For example, when the jugular veins were compressed visual impairment under $G$ was associated with sensations referred to the head, with clonic spasm of the hands, and precordial pain. A beating sensation in ears and head was also not uncommon. Clonic twitching of hands, and individual digits was often experienced on recovering consciousness and sometimes after severe "black-outs", particularly when associated with experiments on the effects of $G$ and anoxia. In one of 50 subjects, however, clonic twitching involving the upper limbs and facial musculature of gross amplitude was observed whilst recovery of consciousness was taking place. The exposure field of the camera unfortunately did not include the lower limbs, but Diringshofen (1941) has stated that generalized convulsions are not uncommon during or after consciousness. Incontinence of urine was noted on one occasion.

A factor of almost equal importance to the actual loss of consciousness was the disturbance in spatial and temporal orientation which was always present in some measure on return to consciousness. It is not known whether this was an intrinsic physiological 
effect, but it has been observed to last from 10-20 second after the erect posture has been regained. The subject was always at a loss to account for changes in orientation on recovery; mental processes were markedly impaired during this period. He might hear the pilot faintly over the intercommunication system, but neither had the will-power nor the understanding to answer. Fully coordinated action, unless of an automatic nature, appears to be impossible.

After effects were similar to, but more marked than in any threshold run. One definite feature has been noticed after unconsciousness, a definite lowering of morale or will-power, normally expressed as a disinclination to undergo further immediate experimentations. If this were necessary, considerable apprehension existed, which was reflected in undue tensing of the muscles, and holding of the breath whilst undergoing the ensuing acceleration.

\section{Physiological Judgment of Value of Acceleration}

The capacity of any one individual to estimate the value of the acceleration may be limited. This is in accordance with the conception of the bombardment of the kinæsthetic sensorium by a flood of stimuli under the great increase in weight. Thus it is diffcult to estimate the value of any amount greater than $4 \mathrm{G}$ to within $1 \mathrm{G}$, especially if the observer be occupied in piloting. It can be stated, however, that $9 \mathrm{G}$ is very different from $6 \mathrm{G}$, and $6 \mathrm{G}$ from $4 \mathrm{G}$. A resistant subject is generally aware of the onset of heavy centrifugal force at about $4 \mathrm{G}$.

\section{Discussion}

There is ample and well-established evidence that the loss of function due to centrifugal force is related to the circulatory effects. It is beyond the scope of this article to attempt any review of the literature, but it is an accepted fact that the visual impairment is primarily retinal in localization. The visual impairment can be considered as a rise in the subjective threshold of the retina and is similar in characteristics to the rise in threshold caused by obstruction of the retinal circulation by external pressure, Craik and Vernon (1941). The latency, between the appearance of ocular symptoms and disturbance of consciousness, is generally ascribed to the intraocular tension, of between $25-30 \mathrm{~mm}$. of mercury, which causes an initial effective decrease in the blood pressure in the central retinal artery as compared to the intracranial pressure. Thus the retina is rendered anoxæmic before the central nervous system (Andina, 1937). There has always been some doubt as to the probable degree of impairment of cortical function when a pilot is " blacked out". Further, it has been thought that the visual impair-

\section{ONSET \& DEVELOPMENT OF UNCONSCIOUSNESS}

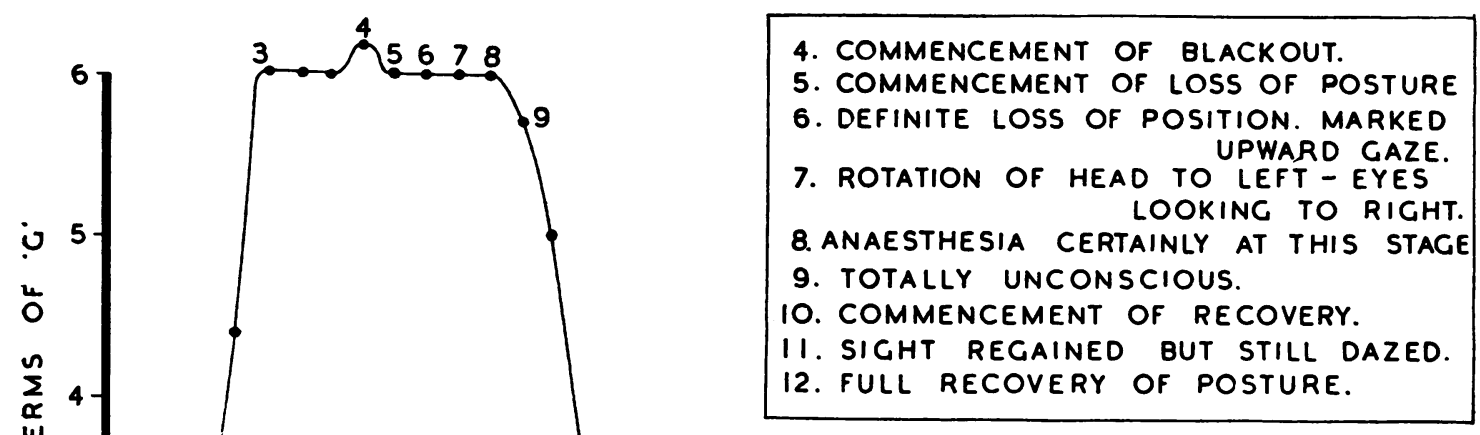


ment may be caused either by pressure upon the optic tracts, due to the increase in weight of the frontal lobes or to occlusion of the posterior cerebral artery, as it crosses the tentorium, by the posterior lobes. The first hypothesis can be dismissed, since it is most unlikely that shearing stresses could be developed in the optic chiasma or tracts, of sufficient degree to produce blockage of the transmission of nervous impulses; and it is known that hydrostatic pressure on the tracts could not produce the visual impairment (Grundfest, 1936). It is, however, difficult to exclude the second hypothesis on evidence at present available. At values of $G$ which do not produce visual symptoms, no reduction in general efficiency can be demonstrated in pilots flying single-seater aircraft, and no impairment of nervous function can be revealed in practical or performance tests which involve flying an aircraft, or in associated duties. However, if low values of $G$ be very frequently repeated in one flight, fatigue does develop during the flight, is experienced during the post-flight period and may"not disappear without rest or sleep. If the flying duties involve continual exposure to low values of $G$ over a period of weeks or months, this type of fatigue may become associated with symptoms of anxiety. At values of $G$ which cause visual impairment, it has been demonstrated that the whole field of vision is affected although the degree of impairment may vary for central or peripheral vision. Owing to the nature of the research, perimetry could not be attempted, but it did appear evident that scotomatous or hemianopic defects were never present. The value of $G$ which, if maintained for 3-5 seconds, results in total visual loss, is termed the "blacking-out", threshold.

It has been demonstrated that, when the pilot is merely "blacked out" at his threshold value, general muscular control is relatively unimpaired, co-ordinated movements are still possible, and the oculomotor apparatus is apparently unaffected. This must indicate, therefore, that there is no major disturbance of the motor, pre-motor, or frontal cortex. General sensation would appear to be intact, although detailed analysis by clinical methods was impossible to carry out. It can also be assumed that there is no marked impairment of the function of the auditory or olfactory cortex. As regards the integrity of the occipital cortex, the voluntary ocular movements did appear to be supported by the fixation reflex in that the eyes could be kept accurately to one side or could be rigidly located on the position of the test card with strong rotation of the head. If the fixation reflex be maintained then there may be no gross impairment of the visual cortex (Holmes, 1938). However, the evidence cannot be taken as conclusive since the duration of the rise in retinal threshold was no more than 12-15 seconds at most. The occurrence of esophoria or of ptosis can be ascribed to latent muscular weakness or imbalance, rather than to paresis.

If the value of centrifugal force be markedly above the individual's threshold, impairment or total loss of consciousness may ensue. The unconsciousness, presumably, is due to cerebral anoxæmia, but disturbance in ionic balance, and fall in glucose content of the cerebral blood vessels may also be partly responsible. If the unconsciousness be due primarily to anoxæmia, one might expect to be able to study selective effects on the general musculature of deprivation of the higher nervous centres of the requisite oxygen. Those neurones which require the greatest oxygen quotient and the greatest vascularity, might be primarily affected. In every instance, however, the primary effect appeared to be loss of posture which occurred before complete paresis of the upper limbs. As has been pointed out above, this.effect may be due to general loss of muscular tone, and the apparent primary location in the trunk musculature may be due to the relative increase in weight under G. Return of consciousness is remarkably rapid and this may not be without physiological significance. As in "blacking out "where return of vision is also dramatically swift, and where complete visual loss is rarely succeeded by a period of veiling, this may indicate that there is no absolute deprivation of blood from retinal or cerebral vessels.

It is obvious that great use can be made of the application of centrifugal force as a means for investigating, not only cortical function in man, but also its dependence upon the biochemical properties of blood and the physical interrelationships of C.S.F., venous and arterial pressures. Likewise a new field of visual investigation is opened up. It is known that the intra-ocular pressure is largely dependent on the general systemic arterial pressure, but that there are normally present mechanisms which tend to modify, or control, the effect of fluctuations in the systemic pressure. The use of centrifugal force presents new opportunities for investigation of this problem.

It has been noted that clonic twitching of the limbs has been observed both in these series of tests and in Diringshofen's series (1941). The frequency of fits in association with unconsciousness under $\mathrm{G}$ cannot be given, owing to the small number of cases (50), and in these complete unconsciousness was only photographed on eleven occasions. However, these clonic fits when present were associated with deep unconsciousness, but not with any abnormalities in the resting encephalogram or with any previous history of fainting.

Lastly, it has been observed that, although there is no gross impairment of cortical function at the "blacking-out" threshold, repeated "blacking out", or exposure to low'values of $G$, does lead to subjective fatigue. The physiological basis of this fatigue is unknown, but it is believed that it may result in part from minor degrees of cortical anoxæmia. There is insufficient increase in oxygen consumption to account for the fatigue on a physical basis, and it is similar to the fatigue which follows collapse on a tilting table. Thus, application of centrifugal force may also be valuable in the study of physiological fatigue. 
Summary

(1) Some observations are presented which support the view that there is minimal impairment of cortical function at the "black-out" threshold.

(2) Application of centrifugal force as a method of experimental investigation holds marked possibilities for the study of fatigue, physiology of vision, of cortical function as well as circulatory problems.

It is a pleasure to thank Air Marshal Sir H. E. Whittingham for permission to publish this article, and Dr. B. H. C. Matthews, F.R.S., for much help and guidance in the research.

\section{REFERENCES}

Andina, F. (1937). Schweiz, med. Wschr., 67, 756.

Burmeister, H. (1939). Luftfahrtmed., 3, 277.

Craik, K. J. W., and Vernon, M. D. (1941). Brit. J. Psychol., 32, 62.

Diringshofen, $\mathrm{H}$. von (1938). Luftfahrtmed., $2,1$. (1939). Medizinischer Leitfaden, für fliegende Besatzungen. Leipzig, Theodor Steinkopff. (1941). Luft fahrtmed., 6, 152.

Grundfest, H. (1936). Cold Harbor Symposia on Quantitative Biology, 4, 179.

Holmes, G. (1938). Brit. med. J., 2, 107.

Livingston, P. C. (1939). Brit. J. Surg., 26, 104.

Rook, A. F., and Dawson, D. J. (1938). Lancet, 1503. 